\title{
ENCRYPTION AND DECRYPTION USING IMAGE PROCESSING TECHNIQUES
}

\author{
R.Uma Maheshwari \\ Department of ECE \\ Hindusthan Institute of Technology, Coimbatore, Tamilnadu, India
}

\begin{abstract}
Images consists various kinds of information which can be used for encryption and decryption of messages through them. This paper proposes a photograph encryption and decryption method to encrypt a covert snapshot with the aid of combining the Arnold transform system within the area and decrypting the duvet photograph through combining inverse Arnold become. First, grow to be a cover snapshot into subparts which consists of eight binary pictures with the aid of decimal value to eight-digit binary operation. Then, change into eight binary snap shots into sub-blocks of eight binary scrambled pixel by means of the Arnold change into, respectively. And then, recombine the sequence of the eight binary scrambled matrices right into a scrambled matrix with 256 grey phases in keeping with the special membership. Discrete Wavelet Transform (DWT) is used to perform picture compression on the input picture and secretly hidden photograph which is finished making use of alpha mixing. Sooner or later, derive an encrypted image from the scrambled photograph with the aid of the Hartley turn out to be. Second, decode the encrypted picture making use of inverse Arnold develop. Inverse DWT is performed to regain the compressed pictures. Simulations indicate that the proposed approach has a bigger photograph scrambling measure, more protection and has the robustness against occlusion and speckle noise attacks.
\end{abstract}

Keywords - Image encryption, Arnold turn out to be, DWT, image decryption, Inverse Arnold transform, Alpha blending, IDWT

\section{INTRODUCTION}

Image encryption ways have two businesses, one encrypts covert snap shots in the house field and another encrypts covert pixel in the frequency field. For the initiative workforce, encrypted covert graphics can be recovered and not using a distortion most likely. Nonetheless, it is much less hindrance of unauthorized persons to decrypt covert images as a rule. Conversely, encrypted covert pictures in the frequency area are with higher protection, parallel and high velocity processing, and it's potent for occlusion attacks and noise assaults. Still, the duvet photographs do not be precisely retrieved ordinarily. This clause will use concurrently two groups of benefits of snapshot encryption ways to encode a covert image. Image scrambling approaches is greatly main photo encrypting methods in the space area. They tin be utilized to increase the robustness of occlusion assaults and noise assaults. They've been divided into two businesses. The starting team is matrix transformations, e.g. The Arnold transforms. They name for proper parameters of the transformation to clear up inverse of the matrix transformation. They have got in result protection; and the decoding method of the scrambled portraits requires keys and the translation are simple. The second staff is coordinating actions. In addition, they need correct parameters of the scrambling route to examine the decoded pictures.

\section{LITERATURE SURVEY}

S. Arivazhagan 1 et al. [1] says that the quite a lot of methods and the various enter photo database which has the various statistical and co-occurrence elements that would be transformed via the Discrete Wavelet Transformer (DWT). However, the classifier used in the present procedure have got to have the very big database but the output is used to have the larger spatial area. The input duvet photo can be encoded and decoded using the DWT and inverse DWT method and it may be performed using alpha mixing. The foremost error within the current work is the increment in the accuracy and the MSE price. Hence the picture has the lesser resolution value. R. Talwar et al. [2] carried out a strong blind watermarking algorithm specifically situated on the algorithm which is used to discrete wavelet become is the Arnold-Chaos encryption and decryption process. The above acknowledged two encryption and decryption methods are used to rent the pixel worth of the photo for the duration of the encryption procedure. Thus, the algorithm used right here is to give the grater worth and the easier way of conversion due to the fact of the values generated by the Arnold and the chaos grow to be. These algorithms will have the various points with the immoderate randomness in the pixel. Zhenjun Tang et al [3] proposed that the encoding method takes location by the Arnold become and the three special ways. The algorithm which is explained above may be very efficient and there's larger accuracy. This proposed algorithm is impartial of size the place it can be used for encryption of any input pixel [4]. Pratibha Sharma et al. [5] mentioned concerning the more than a few three approaches of DWT transform based on image watermarking ways. This proposed procedure can have the 
lesser watermarking result if you want to be invisible and in effective. As a consequence, it can be adopted by way of the alpha mixing to extract the watermarked images and to decrypt the snapshot [6]. The major goal of this process is taking the more than a few numbers of bits and converting the quantity of bits into low frequency sub band with the aid of the alpha mixing., watermark snapshot is dispersing or relying upon the scaling factor of alpha mixing method. The primary stage of the watermarked image is the scaling process which is foremost algorithm utilized in embedding. This framing thought have the various comprehensive services and taking out the encrypted snapshot without lack of knowledge. As a result, the transferring information without lack of information is known as lossless compression. Tian et al. [7] had implemented the exchange sin the pixel as a reversible approach to cover the tremendous portions of knowledge. This reversible process can increase the potential of data to hide the big portions of pixel price. Tsai et al.Eight. [8] presented converted histogram transferring wherein the histogram equalised values and the error weights of histogram which have been located with the aid of the siva Shankar and Rangarajan [9] in the proposed work of knowledge hiding. They had represented 1340 images and the intensity of the photo can also be recognized and valued. Thus, they would create the value within the pixel of the cover photograph. The snapshot pixel values are modified from one bit to another and so they were restrained from zero degrees.

\section{ENCRYPTION APPROACH}

\subsection{Encryption Approach}

First stage of the proposed approach is encryption system which includes encryption procedure

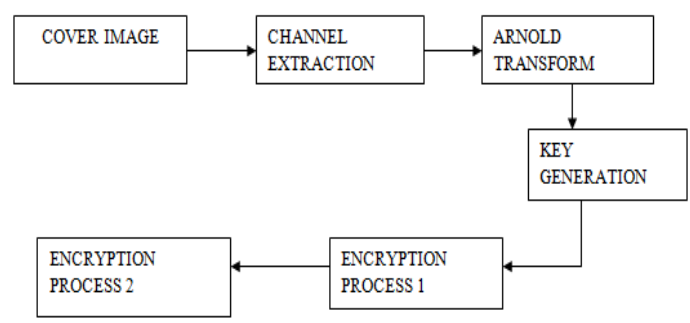

\section{Figure1 Block diagram of encryption approach.}

First step the cover picture is taken as an enter picture and the enter image is loaded manually through the transmitter aspect. Second step is Channel extraction is completed to discover the speckle noise and the compression ratio of the compressed photo using DWT transform which is utilized in encryption approach [10]. Third step is the Arnold turn into process may also be applied to cover the message or to cover the given quilt photograph. Forth process is the key values are generated using non-uniform mapping and the encryption approach is carried out making use of the generated key values [11]
Step1:in the encryption process 1, there would 50\% encoding shall be performed making use of chaotic sequence

Step2:in the encryption method a hundred\% encoding has been implemented.

\subsection{Decryption Approach}

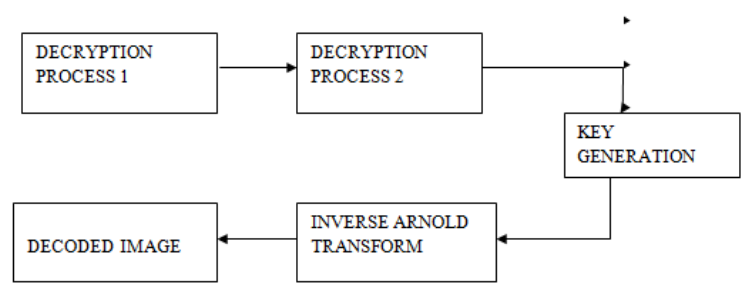

Figure.2 Block diagram of decryption

Block diagram of decryption system second stage of the proposed process is encryption system which consists of more than a few steps as it follows step one follows the decryption method is carried out utilising the inverse Arnold grow to be system following by the chaos mapping. Process two in the decryption system 2 , a hundred\% encrypted image can also be decoded Third process is key values are generated utilizing non-uniform mapping and the decryption system is applied utilizing the generated key values. final is the Inverse Arnold turn out to be approach will also be implemented to put off the message or to unhide the given cover picture

Step1: finally, decoded image may also be an identical closer to the normal snapshot. Thus, the process carried out with the following results.

\section{RESULTS}

Thus, the process is carried out using the biometric images. The biometric images such as Palm, fingerprint is used for the encryption and decryption purposes followed by the compression process. The following figures shows the procedure of encryption and decryption in biomedical images

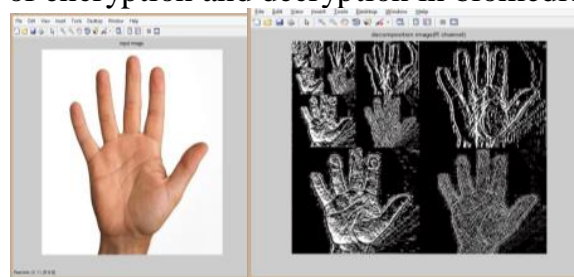

Figure 3 Original image Figure 4 Pre-processed image

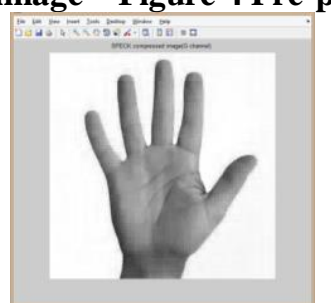

Figure 5 Compressed image 
International Journal of Engineering Applied Sciences and Technology, 2021

Vol. 5, Issue 12, ISSN No. 2455-2143, Pages 219-222

Published Online April 2021 in IJEAST (http://www.ijeast.com)

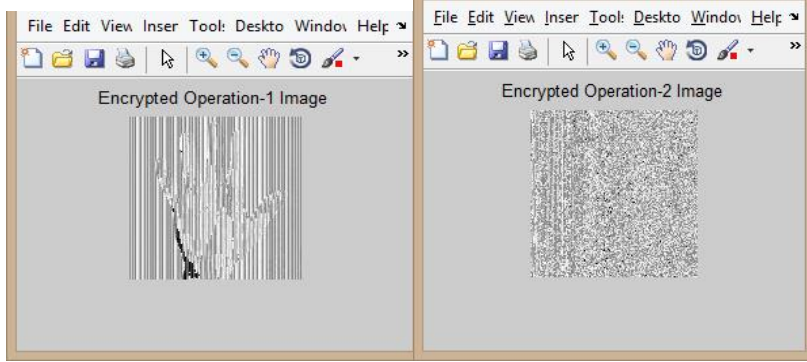

Figure 6 Encrypted image 1 Figure 7 Encrypted image 2

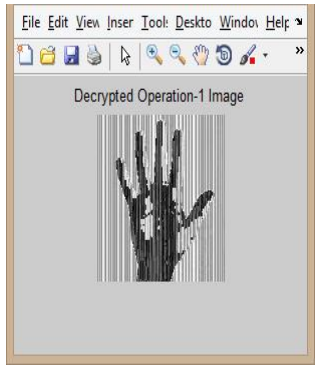

Figure 8Decrypted image 1

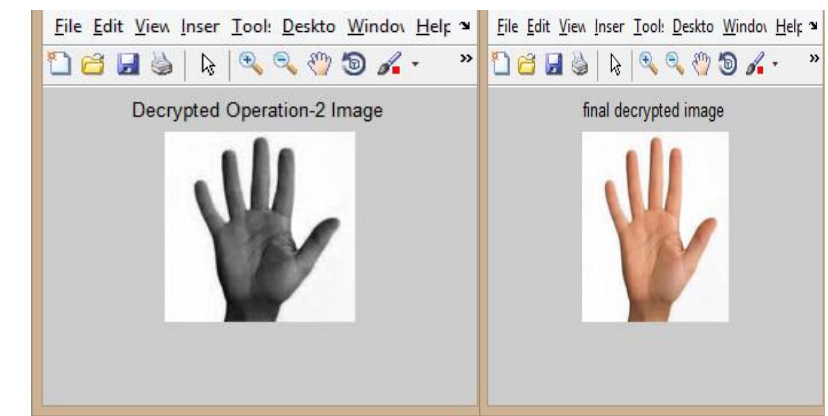

Figure 9 Decrypted image 2 image

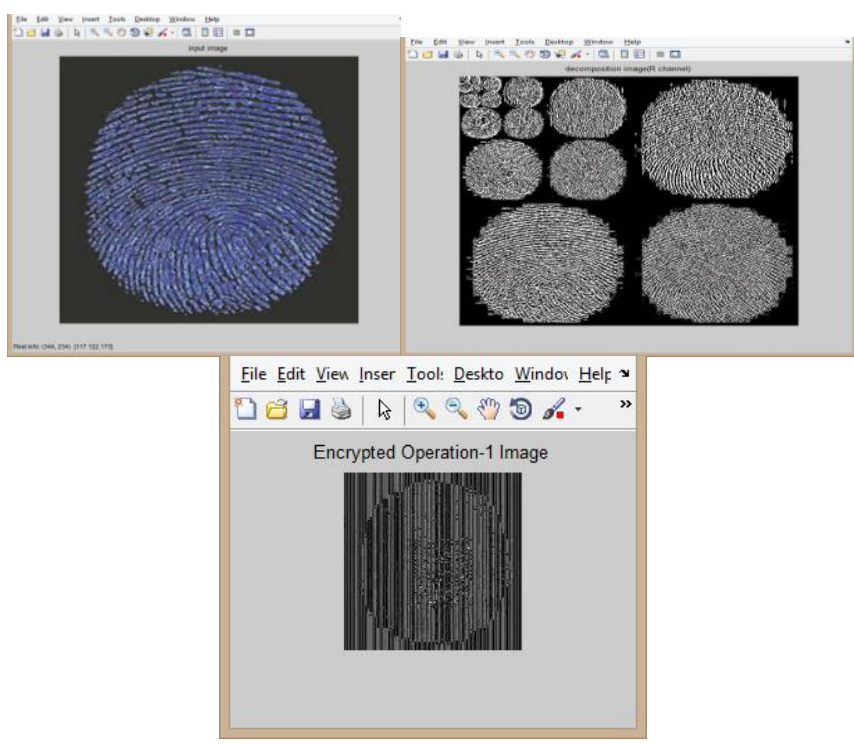

Figure 11 original image Figure 12 Pre-processed image Figure 13 Encrypted image 1

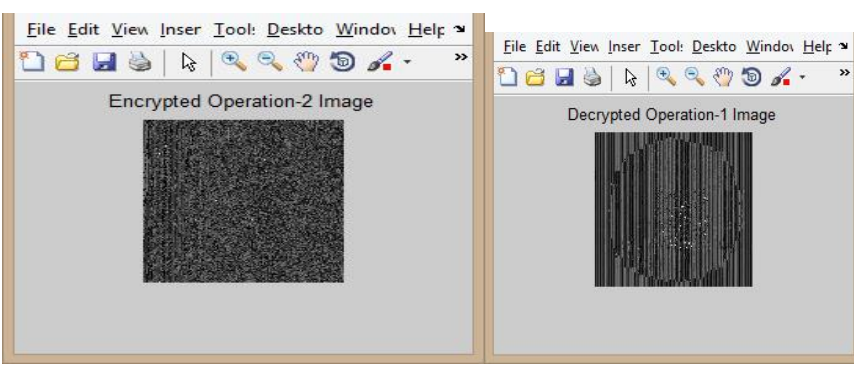

Figure 14 Encrypted image 2 Figure 15 Decrypted image 1

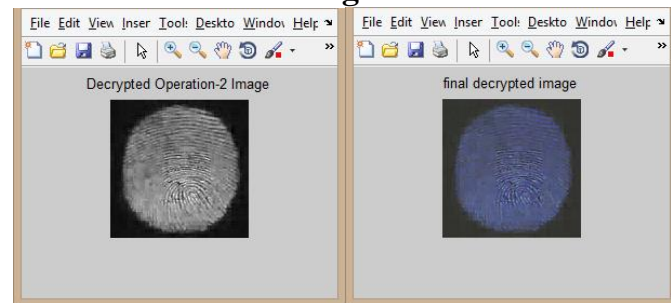

Figure 16 Decrypted image 2 Figure 17 Final image

\section{PERFORMANCE MEASURE}

Thus, the performance measure indicates quality of the compressed encrypted and decrypted images. This performance measure is shown in the table 1

Table 1 Performance metrics 


\begin{tabular}{|l|l|l|l|l|}
\hline ACCURACY & SENSITIVTIY & PSNR & MSE & RMSE \\
\hline 86 & 76.234 & 25.234 & 56.28 & 47.96 \\
\hline 90 & 85.121 & 23.456 & 45.23 & 89.53 \\
\hline
\end{tabular}

\section{REFERENCE}

[1] Zhang, W., Wong, K. W., Yu, H., \& Zhu, Z. L. (2013). A symmetric color image encryption algorithm using the intrinsic features of bit distributions. Communications in Nonlinear Science and Numerical Simulation, 18(3), 584-600. [2] Yoon, J. W., \& Kim, H. (2010). An image encryption scheme with a pseudorandom permutation based on chaotic maps. Communications in Nonlinear Science and Numerical Simulation, 15(12), 3998-4006.

[3] Zhang, G., \& Liu, Q. (2011). A novel image encryption method based on total shuffling scheme. Optics Communications, 284(12), 2775-2780.

[4] Wang, X. Y., Gu, S. X., \& Zhang, Y. Q. (2015). Novel image encryption algorithm based on cycle shift and chaotic system. Optics and Lasers in Engineering, 68, 126-134.

[5] Wang, X., \& Luan, D. (2013). A novel image encryption algorithm using chaos and reversible cellular automata. Communications in Nonlinear Science and Numerical Simulation, 18(11), 3075-3085.

[6] Enayatifar, R., Sadaei, H. J., Abdullah, A. H., Lee, M., \& Isnin, I. F. (2015). A novel chaotic based image encryption using a hybrid model of deoxyribonucleic acid and cellular automata. Optics and Lasers in Engineering, 71, 33-41.

[7] Wang, L., Song, H., \& Liu, P. (2016). A novel hybrid color image encryption algorithm using two complex chaotic systems. Optics and Lasers in Engineering, 77, 118-125.

[8] Liu, H., \& Kadir, A. (2015). Asymmetric color image encryption scheme using 2D discrete-time map. signal processing, 113, 104-112.

[9] Wu, X., Kan, H., \& Kurths, J. (2015). A new color image encryption scheme based on DNA sequences and multiple improved 1D chaotic maps. Applied Soft Computing, 37, 2439.

[10] Zhou, N., Zhang, A., Zheng, F., \& Gong, L. (2014). Novel image compression-encryption hybrid algorithm based on key-controlled measurement matrix in compressive sensing. Optics \& Laser Technology, 62, 152-160.

[11] Xu, L., Li, Z., Li, J., \& Hua, W. (2016). A novel bit-level image encryption algorithm based on chaotic maps. Optics and Lasers in Engineering, 78, 17-25.

[12] Belazi, A., Abd El-Latif, A. A., Diaconu, A. V., Rhouma, R., \& Belghith, S. (2017). Chaos-based partial image encryption scheme based on linear fractional and lifting wavelet transforms. Optics and Lasers in Engineering, 88, 3750 .
[13] Chai, X., Chen, Y., \& Broyde, L. (2017). A novel chaos-based image encryption algorithm using DNA sequence operations. Optics and Lasers in engineering, 88, 197-213.

[14 ] Abdo, A. A., Lian, S., Ismail, I. A., Amin, M., \& Diab, H. (2013). A cryptosystem based on elementary cellular automata. Communications in Nonlinear Science and Numerical Simulation, 18(1), 136-147. 